\title{
COVID-19 autopsy cases: detection of virus in endocrine tissues
}

\author{
A. M. Poma ${ }^{1}$ - D. Bonuccelli ${ }^{2} \cdot$ R. Giannini ${ }^{1}$ - E. Macerola ${ }^{1} \cdot$ P. Vignali ${ }^{1}$. C. Ugolini ${ }^{1} \cdot$ L. Torregrossa $^{1}$ - A. Proietti ${ }^{1}$. \\ M. Pistello ${ }^{3} \cdot$ A. Basolo $^{4} \cdot$ F. Santini ${ }^{4} \cdot$ A. Toniolo ${ }^{5} \cdot$ F. Basolo $^{1}$ (I)
}

Received: 7 June 2021 / Accepted: 25 June 2021 / Published online: 30 June 2021

(C) Italian Society of Endocrinology (SIE) 2021

\begin{abstract}
Purpose The SARS-CoV-2 genome has been detected in a variety of human samples including blood, urine, semen, and faeces. However, evidence of virus presence in tissues other than lung are limited.

Methods We investigated whether SARS-CoV-2 could be detected in 50 autoptic specimens of endocrine organs from 29 patients who died of COVID-19.

Results The virus was detected in 25 specimens including ten abdominal subcutaneous adipose tissue samples (62\%), six testes $(67 \%)$, and nine thyroid (36\%) samples. The analysis of multiple endocrine organ samples obtained from the same patients showed that, in virus-positive cases, the viral genome was consistently detected in all but two matched specimens. Conclusion Our findings show that the virus spread into endocrine organs is a common event in severe cases. Further studies should assess the rate of the phenomenon in clinically mild cases. The potential long-term effects of COVID-19 on endocrine functions should be taken into consideration.
\end{abstract}

Keywords SARS-CoV-2 $\cdot$ COVID-19 $\cdot$ Adipose tissue $\cdot$ Testis $\cdot$ Thyroid

\section{Introduction}

The Severe Acute Respiratory Syndrome Coronavirus 2 (SARS-CoV-2) is the causative agent of the Coronavirus Disease 2019 (COVID-19). As of June 7, 2021, more than 173 million cases have been reported worldwide with over 3.7 million deaths. (https://www.arcgis.com/apps/opsda shboard/index.html\#/bda7594740fd40299423467b48e9ec f6).

F. Basolo

fulvio.basolo@med.unipi.it

1 Department of Surgical, Medical, Molecular Pathology and Critical Area, University of Pisa, Via Savi,10, 56126 Pisa, Italy

2 Department of Forensic Medicine, Azienda USL Toscana Nordovest, Lucca, Italy

3 Retrovirus Center and Virology Section, Department of Translational Research and New Technologies in Medicine and Surgery, University of Pisa, Pisa, Italy

4 Department of Clinical and Experimental Medicine, University of Pisa, Pisa, Italy

5 Global Virus Network, University of Insubria, Varese, Italy
As indicated by the exceptionally high rate of pneumonia in moderate-to-severe cases, the primary target of SARSCoV-2 is the lung [1]. However, COVID-19 is a systemic disease that can hit several different organs [2]. Indeed, the SARS-CoV-2 receptor angiotensin-converting enzyme 2 (ACE2) and co-receptor Transmembrane Serine Protease 2 (TMPRSS2) are expressed in a variety of tissues and also in endocrine organs [3-5]. Accordingly, the infection may be associated with endocrine conditions. For instance, about $20 \%$ of hospitalized COVID-19 patients develop thyrotoxicosis [6]. Cases of subacute thyroiditis have also been observed, suggesting that the virus could directly infect thyroid cells [7, 8]. SARS-CoV-2 has been detected in the semen of six COVID-19 patients (about $16 \%$ of individuals tested), including two during the recovery phase. Consequently, infection of the male genital tract is likely to occur, although the presence of the virus in testes has yet to be demonstrated [9]. Finally, among the predictors of poor outcome such as aging, male sex, diabetes, and hypertension, also overweight and obesity seem to play a central role. This may be due to an enhanced immune activation and cytokine production in obese patients [10], but it has been suggested that the adipose tissue (which communicates with other tissues to regulate the systemic metabolism through secretion 
of adipocyte-derived peptide hormones, inflammatory mediators, signalling lipids, and microRNAs [11]) might be involved in virus tropism and replication [12].

\section{Materials and methods}

Fifty endocrine FFPE tissue samples obtained from 29 patients and 18 non-COVID-19 control cases were tested for the SARS-CoV-2 genome by means of an RT-PCR assay targeting the viral nucleocapsid (N) and RNA-dependent RNA polymerase (RdRp) genes (Easy SARS-CoV-2 WE kit, Diatech Pharmacogenetics, Jesi, Italy). In details, the following specimens were tested in the COVID-19 cohort: 16 abdominal subcutaneous adipose tissues, 9 testes, and 25 thyroid specimens. These were compared to non-COVID-19 control cases: 4 abdominal subcutaneous adipose tissues, 3 testes, and 11 thyroid specimens.

RNA was isolated from samples using the RNeasy FFPE kit (Qiagen, Hilden, Germany). Viral assays were run in duplicate using about $250 \mathrm{ng}$ total RNA per test. Samples were deemed positive when amplicons were obtained for the $\mathrm{N}$ or the RdRp gene, or for both. Borderline results were retested using a different RNA sample extracted from the same tissue. Relative expression levels were computed as function of the cycle threshold $(\mathrm{Ct})$ according to the $2^{-\Delta \Delta \mathrm{Ct}}$ method using the RT-PCR internal positive control as reference.

To check whether SARS-CoV-2 antigens could be detected in the adipose tissue, testis, and thyroid, threemicrometer-thick sections were stained with anti-SARS nucleocapsid protein antibody (NB100-56683, Novus Biologicals, Centennial, CO, United States) using an automated staining system (BenchMark ULTRA, Ventana Medical Systems, Oro Valley, AZ, United States). Antibody binding was evidenced with the OptiView DAB IHC Detection Kit (Ventana). Slides were counterstained with Hematoxylin II and Bluing Reagent (Ventana).

All analyses and plots were generated in R environment (https://www.r-project.org, v.4.0.2, last accessed April 2021).

The study was approved by the regional scientific ethics committee.

\section{Results}

The SARS-CoV-2 genome was searched in endocrine specimens of 29 autopsy cases of COVID-19 (median age 66 years, IQR 42-74; 23 males, 6 females) as well as in 18 autopsy cases of individuals who died for causes other than COVID-19 (median age 60 years, IQR 48-71; 8 males, 10 females). The median time from initial symptoms to death was 7 days (range 1-51). The median time from death to autopsy was 5 days (range 1-30). All COVID-19 cases presented histopathologic changes of lung consistent with moderate-to-severe COVID-19.

In all cases of the COVID-19 cohort, SARS-CoV-2 was detected both pre-mortem (nasopharyngeal swabs) and postmortem (lung tissue). Comorbidities were present in 25/29 (86\%) COVID-19 cases: 12 cardiovascular disease (41\%), 10 overweight or obesity (34\%), 7 diabetes (24\%), 4 chronic pulmonary disease (14\%), and 3 malignancy (10\%). No evidence of the SARS-CoV-2 genome was found in the control cases (post-mortem nasopharyngeal swab, lung, adipose, testis, and thyroid tissues).

The SARS-CoV-2 genome was detected in 10/16 (62\%) of adipose tissue samples, 6/9 (67\%) of testes, and 9/25 (36\%) of thyroid specimens. Figure 1 shows the relative quantification of SARS-CoV-2N and RdRp gene amplicons in the adipose tissue (A), testis (B), and thyroid (C). Viral genome levels ranged widely among cases, with the adipose tissue bearing the lowest levels, and the testis and thyroid showing the highest levels. Figure 1D shows data of 10 cases from which more than one organ was sampled. The time from the initial symptoms to death (as referred by family members and hence likely inferior to the real length of symptoms) ranged from 1 to 15 days. In 8/10 cases, virus was concordantly detected in two or three tissue types. Discordant results were obtained for $2 / 10$ cases. The two latter cases, however, were characterized by extremely low virus loads.

Immunohistochemistry showed staining for the SARSCoV-2 nucleocapsid antigen in all cases that were positive by RT-PCR. However, the intensity and the extent of staining was variable from focal positivity to large geographic staining. Notably, the degree of immunostaining was not always concordant with the viral load as measured by RTPCR. In details, in a small proportion of adipose cells, the thin rim of cytoplasm surrounding large lipid droplets stained positive for the nucleocapsid coronavirus antigen (Fig. 2B). Similarly, cytoplasmic nucleocapsid staining was observed in cells of seminiferous tubuli in testes (Fig. 2D) and in the epithelial cells lining thyroid follicles (Fig. 2F). Thus, in patients who died of COVID-19 but not in controls, viral proteins of SARS-CoV-2 were expressed in cells of multiple endocrine organs.

\section{Discussion}

In the COVID-19 cohort, the SARS-CoV-2 genome was detected in 25/50 (50\%) endocrine specimens.

Hence, the virus spreading into endocrine organs emerges as a common event in COVID-19.

However, positivity of PCR assays might also represent viral spreading via blood/lymphatics rather than specific virus tropism [2]. Immunohistochemistry indicates that 

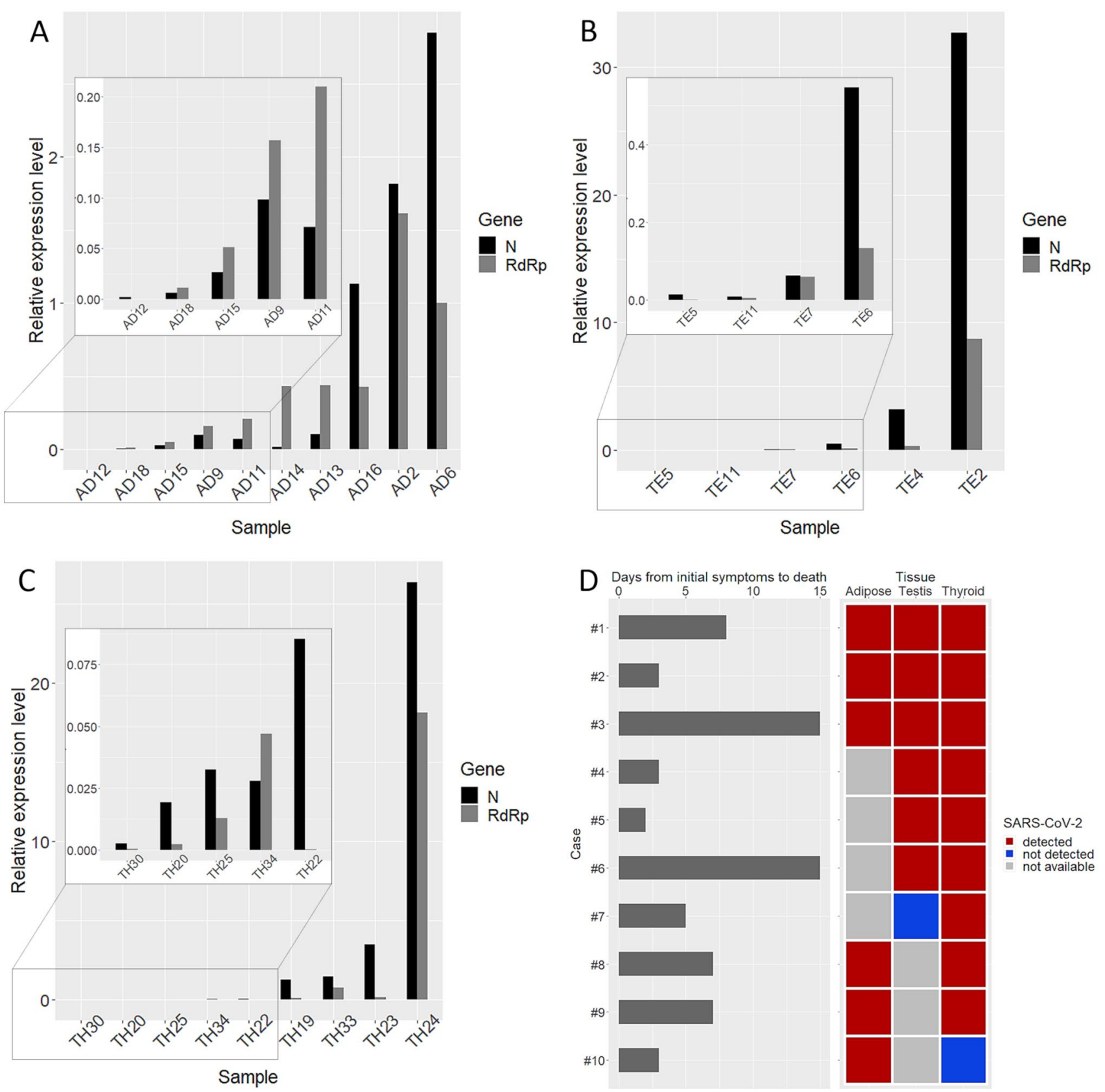

Fig. 1 The bar plots show the relative abundance of SARS-CoV-2 nucleocapsid (N) and RNA-dependent RNA polymerase (RdRp) gene amplicons in adipose tissue (A), testis $(\mathbf{B})$, and thyroid (C). Relative expression levels were computed by the $2^{-\Delta \Delta \mathrm{Ct}}$ method using the RTPCR internal positive control as reference. Insects refer to cases with

virus replication does occur in differentiated cells of multiple endocrine tissues. It should be reminded that the viral tropism is influenced by genetic diversity [13], and that variation is implicit in clinical studies.

The demonstration that SARS-CoV-2 may directly infect the adipose tissue, testis, and thyroid helps explain the previous clinical findings [6-9]. Subacute thyroiditis has very low viral load that were not visible using the default scale. D Ten cases for which different organs were available. Left: grey bars represent days from initial symptoms to death as reported by family members. Right, detection of SARS-CoV-2 genome in matched samples (red, positive; blue, negative; grey, specimen not available)

been reported in patients with COVID-19, and, typically, the condition is considered of viral origin [14]. We did not observe morphological alterations associated with virus positivity. The onset of thyroid conditions after COVID-19 is typically delayed, and can even occur after disease resolution. In our cases, the great majority of subjects died in less than 2 weeks from symptoms presentation. Consequently, 


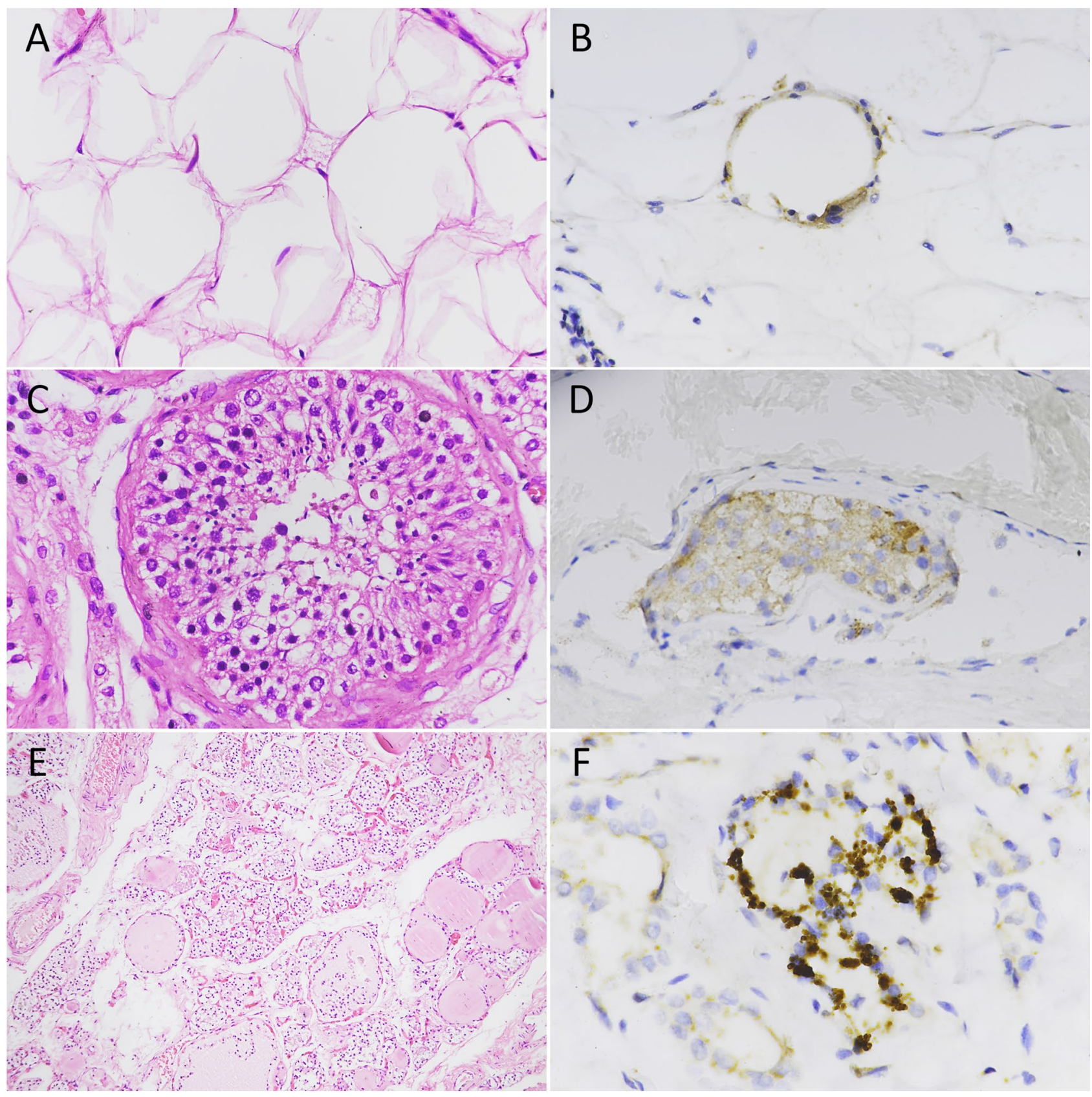

Fig. 2 Hematoxylin and eosin staining of adipose, testis, and thyroid specimens $(\mathbf{A}, \mathbf{C}, \mathbf{E})$ and immunohistochemical staining of SARSCoV-2 nucleocapsid antigen $(\mathbf{B}, \mathbf{D}, \mathbf{F})$. A Histologic sections of adipose tissue showing adipocytes of different size. B Granular distribution of SARS-CoV-2 nucleocapsid antigen in adipose tissue. C High magnification of normal testis showing seminiferous tubuli. D

we cannot exclude that the absence of plain and consistent parenchyma alterations is due to the short period from infection to death.

SARS-CoV-2 has been detected also in semen, but the biological basis of the phenomenon is unclear. Our results show that the nucleocapsid antigen of SARS-CoV-2
Moderate brown staining of SARS-CoV-2 nucleocapsid antigen in the cytoplasm of testis cells. E Low magnification of thyroid parenchyma. Follicles of different size are present. F Strong cytoplasmic staining for SARS-CoV-2 nucleocapsid antigen in thyroid follicular cells. Original magnification: A $40 \times$, B $40 \times$, C $40 \times$, D $40 \times$, E $10 \times$, F $60 \times$

accumulates in the cytoplasm of seminiferous cells, an indication of specific viral tropism for the male reproductive tract.

Finally, the virus was frequently detected in the adipose tissue, supporting the contention that the adipose tissue represents a key target for virus replication and the 
associated inflammatory changes [12]. Therefore, the poor prognosis of COVID-19 in overweight/obese subjects [15] may be due not only to the comorbidities and immune alterations typical of the above patients, but also to a direct effect of the virus within adipocytes.

It should be acknowledged that all tested cases had moderate-to-severe disease, and that virus spread is likely to be reduced in mild clinical conditions. In addition, the post-mortem interval before sampling could affect RNA quality and, consequently, the subsequent assays. Nevertheless, evidences support that viral nucleocapsid antigens are strongly resistant to proteolysis $[16,17]$ and that RNA is surprisingly resistant to post-mortem degradation [18]. Also, the deterioration of RNA is tissue-specific, gene-specific, and even genotype-dependent [19]. In our study, we did not observe significant differences in the post-mortem interval according to the immune or molecular detection of virus in endocrine tissues. Moreover, we detected the SARS-CoV-2 genome and antigens in cases with a post-mortem interval of up to 30 days, which is our maximum range.

Since an autopsy is the only opportunity to observe the organs and take optimal samples, these types of studies should be encouraged and should be complemented by taking biopsy specimens when clinically allowed. Investigating which organs are infected by SARS-CoV-2 at different stages of the disease is of high significance for designing effective cures and for monitoring the long-term effects of this dreadful pandemic.

Acknowledgements We would like to thank C. Niccoli, S. Nuti, S. Pelliccioni, and M. Urpi Castany (University Hospital of Pisa) for expert assistance in histology and immunohistochemistry.

Authors' contributions AMP, DB, RG, FS, AT, and FB conceived and designed the study; AMP, DB, EM, PV, CU, LT, AP, MP, and AB participated in data collection; AMP and RG participated in data analysis; AMP, AT, and FB contributed to the interpretation of the results and in the writing the manuscript. All authors participated in the revision of the manuscript.

Funding This work was supported by the University of Pisa.

Availability of data and materials Data sharing is not applicable to this article as no datasets were generated or analysed during the current study.

Code availability Not applicable.

\section{Declarations}

Conflict of interest All authors have no conflict of interest to declare.

Ethics approval The study was performed in accordance with the Declaration of Helsinki and its later amendments, and it was approved by the regional scientific ethics committee (protocol number 17327; May $14,2020)$.
Consent to participate Written informed consent was obtained from the parents.

Consent for publication Written informed consent was obtained from the parents.

\section{References}

1. Huang C, Wang Y, Li X et al (2020) Clinical features of patients infected with 2019 novel coronavirus in Wuhan, China. Lancet 395:497-506. https://doi.org/10.1016/S0140-6736(20)30183-5

2. Peng L, Liu J, Xu W et al (2020) SARS-CoV-2 can be detected in urine, blood, anal swabs, and oropharyngeal swabs specimens. J Med Virol 92:1676-1680. https://doi.org/10.1002/jmv.25936

3. Li M-Y, Li L, Zhang Y, Wang X-S (2020) Expression of the SARS-CoV-2 cell receptor gene ACE2 in a wide variety of human tissues. Infect Dis Poverty 9:45. https://doi.org/10.1186/ s40249-020-00662-x

4. Müller JA, Groß R, Conzelmann C et al (2021) SARS-CoV-2 infects and replicates in cells of the human endocrine and exocrine pancreas. Nat Metab 3:149-165. https://doi.org/10.1038/ s42255-021-00347-1

5. Muus C, Luecken MD, Eraslan G et al (2021) Single-cell meta-analysis of SARS-CoV-2 entry genes across tissues and demographics. Nat Med 27:546-559. https://doi.org/10.1038/ s41591-020-01227-z

6. Lania A, Sandri MT, Cellini M et al (2020) Thyrotoxicosis in patients with COVID-19: the THYRCOV study. Eur J Endocrinol 183:381-387. https://doi.org/10.1530/EJE-20-0335

7. Brancatella A, Ricci D, Viola N et al (2020) Subacute thyroiditis after Sars-COV-2 infection. J Clin Endocrinol Metab. https://doi. org/10.1210/clinem/dgaa276

8. Muller I, Cannavaro D, Dazzi D et al (2020) SARS-CoV-2-related atypical thyroiditis. Lancet Diabetes Endocrinol 8:739-741. https://doi.org/10.1016/S2213-8587(20)30266-7

9. Li D, Jin M, Bao P et al (2020) Clinical characteristics and results of semen tests among men with coronavirus disease 2019. JAMA Netw Open 3:e208292. https://doi.org/10.1001/jamanetworkopen. 2020.8292

10. Pasquarelli-do-Nascimento G, Braz-de-Melo HA, Faria SS et al (2020) Hypercoagulopathy and adipose tissue exacerbated inflammation may explain higher mortality in COVID-19 patients with obesity. Front Endocrinol (Lausanne) 11:530. https://doi.org/10. 3389/fendo.2020.00530

11. Kahn CR, Wang G, Lee KY (2019) Altered adipose tissue and adipocyte function in the pathogenesis of metabolic syndrome. $\mathrm{J}$ Clin Invest 129:3990-4000. https://doi.org/10.1172/JCI129187

12. Ryan PM, Caplice NM (2020) Is adipose tissue a reservoir for viral spread, immune activation, and cytokine amplification in coronavirus disease 2019? Obesity (Silver Spring) 28:1191-1194. https://doi.org/10.1002/oby.22843

13. Latini A, Agolini E, Novelli A et al (2020) COVID-19 and genetic variants of protein involved in the SARS-CoV-2 entry into the host cells. Genes (Basel). https://doi.org/10.3390/genes11091010

14. Desailloud R, Hober D (2009) Viruses and thyroiditis: an update. Virol J 6:5. https://doi.org/10.1186/1743-422X-6-5

15. Cai S-H, Liao W, Chen S-W et al (2020) Association between obesity and clinical prognosis in patients infected with SARSCoV-2. Infect Dis Poverty 9:80. https://doi.org/10.1186/ s40249-020-00703-5

16. Li Y, Bourlet T, Andreoletti L et al (2000) Enteroviral capsid protein VP1 is present in myocardial tissues from some patients with 
myocarditis or dilated cardiomyopathy. Circulation 101:231-234. https://doi.org/10.1161/01.cir.101.3.231

17. Longhi S, Receveur-Bréchot V, Karlin D et al (2003) The C-terminal domain of the measles virus nucleoprotein is intrinsically disordered and folds upon binding to the C-terminal moiety of the phosphoprotein. J Biol Chem 278:18638-18648. https://doi.org/ 10.1074/jbc.M300518200

18. Fordyce SL, Kampmann M-L, van Doorn NL, Gilbert MTP (2013) Long-term RNA persistence in postmortem contexts. Investig Genet 4:7. https://doi.org/10.1186/2041-2223-4-7
19. Zhu Y, Wang L, Yin Y, Yang E (2017) Systematic analysis of gene expression patterns associated with postmortem interval in human tissues. Sci Rep 7:5435. https://doi.org/10.1038/ s41598-017-05882-0

Publisher's Note Springer Nature remains neutral with regard to jurisdictional claims in published maps and institutional affiliations. 